\title{
Development of an information system structure for photo-video recording of traffic violations
}

\author{
Marina Bolsunovskaya ${ }^{1}$, Alexander Leksashov ${ }^{1}$, Svetlana Shirokova ${ }^{1, *}$, and \\ Vladimir Tsygan ${ }^{1}$ \\ ${ }^{1}$ Peter the Great St. Petersburg Polytechnic University, Polytechnicheskaya, 29, 195251, Saint- \\ Petersburg, Russian Federation
}

\begin{abstract}
The development of road transport has a positive impact on society: freight and passenger transport are carried out, and communication between cities is improving. But as cars increase, the speed and intensity of traffic increases. And this affects the growth of traffic violations. About $90 \%$ of accidents occur due to the fault of drivers of vehicles that do not comply with the speed limit, as well as the rules of travel. Automatic detection systems for traffic violations have been recognized all over the world and have proven their effectiveness. But there is a need to develop systems for recording violations based on uniform and clear requirements. This will reduce the number of low-quality hardware and related software. The work is devoted to the development of the functional structure of the information system for photo-video recording of traffic violations based on common requirements and standards.
\end{abstract}

\section{Introduction}

The development of road transport has a positive impact on society: the system of communication between different settlements is improved, freight and passenger transportation is carried out through the vehicle, etc. However, an increase in the car fleet also has a negative effect - the speed and intensity of vehicle traffic on roads increases significantly, which entails various kinds of violations of traffic rules.

As we know, road accidents are one of the most serious socio-economic problems of our time. She acquired this sharpness for the following reasons:

- non-compliance of the road transport infrastructure with requirements for safe traffic;

- insufficient effectiveness of the functioning of the security system;

- low level of legal culture of road users;

- low level of driver training for vehicle drivers;

- imperfection of technical means of traffic management [1,2].

According to statistics from the Ministry of Internal Affairs, about 18 thousand people die in accidents each year, and the number of victims reaches 200 thousand. Most of these

\footnotetext{
*Corresponding author: swchirokov@mail.ru
} 
people, the able-bodied population that was involved in production, paid taxes to the state. Thus, new demographic and social problems in society appear.

It is also worth noting that about $90 \%$ of accidents occur precisely due to the fault of drivers of vehicles who do not comply with the high-speed regime, as well as the rules for passing intersections. The suppression of these types of violations is the main task of the traffic police.

According to the results of studies, it was found that in reality the number of violations is much higher (2-2.5 times). This is due to the fact that some of them are not recorded in any way - road accident participants receive verbal warnings or "pay off" from inspectors through bribes. Such negligence of employees subsequently entails a feeling of impunity among drivers.

The state is taking various measures to reduce traffic accidents - the legislative system in the field of traffic is being optimized, changes are being made in the system of governing bodies. Also, one of the main tasks of the state today is the introduction of systems for automatically recording violations of traffic rules. These funds have gained worldwide recognition and have proven effective - the number of accidents in some regions has decreased by $22 \%$. However, the lack of uniform and clear requirements for the created fixation systems leads to an increase in the number of low-quality equipment and related software.

In connection with the above aspects, the topic of research work is relevant.

The aim of this work is to develop the functional structure of the photo-video information system for fixing traffic violations.

To achieve this goal in the course of work, the following tasks must be solved:

1. domain analysis;

2. a comparative analysis of existing IP;

3. the rationale for the implementation of IP;

4. a description of models of existing business processes;

5. determination of requirements for the developed system;

6. development of a functional structure;

7. a description of the functions and tasks of the complex;

8. assessment of the effect of the implementation of the developed system [1-3].

\section{Materials and methods}

On July 1, 2008, Russia began the active introduction of automatic photo-video recording systems that allow real-time monitoring of the situation on the roads and timely detection of offenders. Unlike traffic police officers, fixation cameras can work around the clock, both in daylight and in the dark, filtering the flow of vehicles. For an hour of work, the inspector can fix about 5 vehicles, while the automatic complex will fix all intruders in the field of vision.

Every year, the number of cameras of video-video recording on the road increases and is already $\approx 15$ thousand. With the help of such automatic systems, the level of safety on the road is increased by forcing drivers to comply with traffic rules. To date, they have issued over 80 million administrative offenses.

From the figures 1 and 2, it can be concluded that with the increase in the use of automatic complexes for fixing traffic violations, the number of accidents decreases, and as a result, the number of dead people. According to the results of 2019, about 164 thousand traffic accidents occurred in the country, in which 211 thousand people were injured, and the death toll was 17 thousand. Compared to 2015, the number of accidents decreased by $11 \%$, and the mortality rate by $27 \%$. 


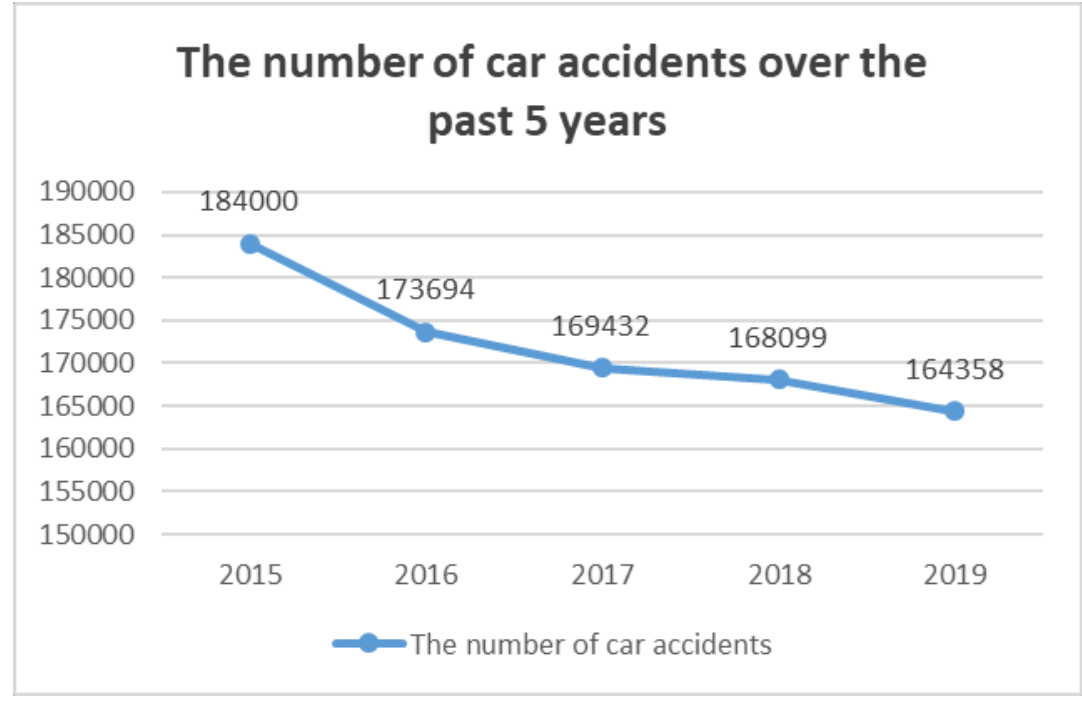

Fig. 1. The number of car accidents over the past 5 years.

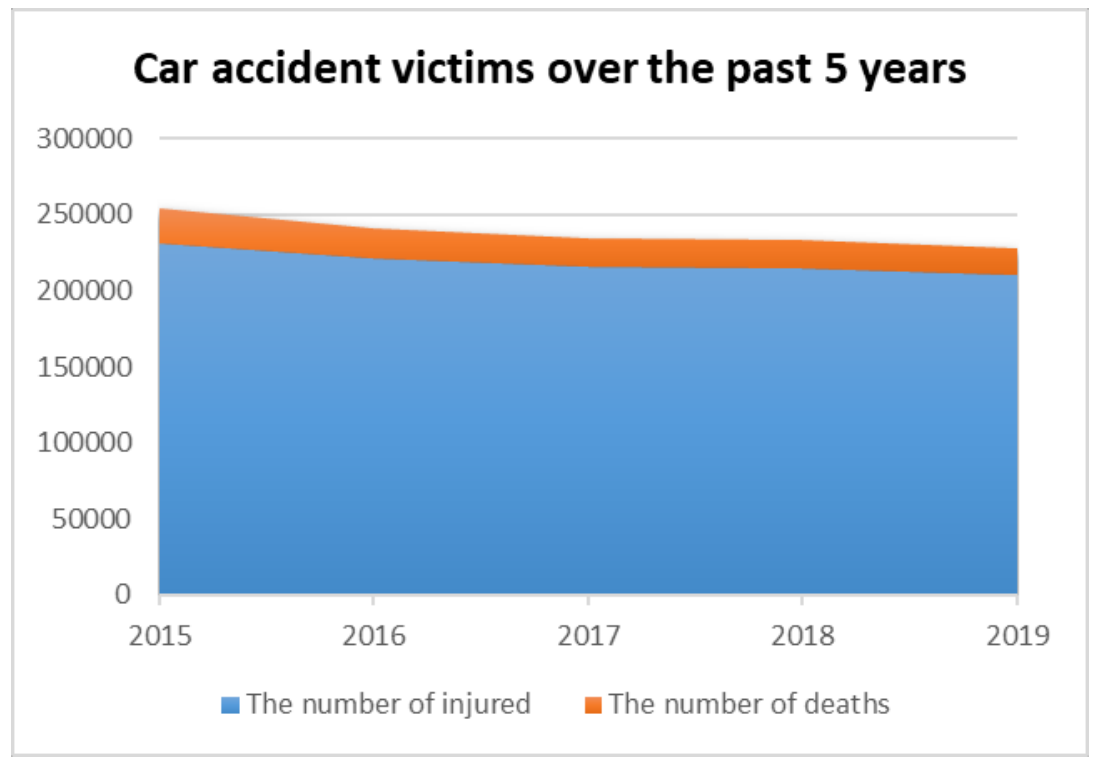

Fig. 2. The number of car accident victims over the past 5 years.

Complexes usually include technical means (photo radar sensors, data transmission equipment, routers, etc.), as well as software that analyzes the resulting video materials. Photo radar sensors, in turn, consist of a radar that detects vehicle speed, and cameras with high sensitivity. The information received through the cameras is processed according to the algorithms set in the software, analyzed and, if it meets certain conditions, is recorded as a violation.

Tasks solved by complexes:

- providing round-the-clock control of traffic on the roads;

- objective assessment of violations;

- reduction in the number of traffic accidents;

- automation of the process of detecting offenses. 
Functions of fixing systems [4,5]:

- determination of the presence in the field of view of the cameras of vehicles and pedestrians, as well as their number;

- fixing the speed and trajectory of vehicles;

- determination of state license plates of vehicles;

- monitoring the intersection of road markings;

- determination of the facts of stopping vehicles, etc.

Complexes of photo-video fixation can be classified according to several criteria $[4,5]$ :

1.Depending on the installation method.

a) stationary complexes - fixed (permanently located in the same place) complexes located over sections of roads at various structures;

b)mobile complexes - mobile complexes placed on the side of the road on special tripods;

c) mobile complexes - quick response complexes placed on public transport and vehicles of traffic police officers to work in automatic mode or used directly by the inspector in manual mode.

2.Depending on the method of fixing violations.

a) Radar - complexes consisting of a radar sensor and the camera's eye directly. At the first stage, the system measures the speed of vehicles using radar and Doppler beam technology, then the camera photographs the registration number of the car, which is then recognized by a special program.

b)Video recordings are complexes that use materials from cameras to measure speed. Photos and videos are transferred to a computer, where the software already uses special algorithms to calculate the speed of the car.

c) Laser - are complexes equipped with cameras with two lenses, one of which is a laser meter, and the other is a camera that records violations.

3. By the method of measuring vehicle speed.

- measuring instantaneous speed,

- calculating the average speed,

- combined.

At the moment, systems record 9 main types of violations:

1.Violation of the speed limit. One of the first violations recorded by cameras. Most often, complexes aimed at suppressing this violation are located on suburban roads, in settlements, as well as on their borders. Exceeding speed by $20-40 \mathrm{~km} / \mathrm{h}$ is punishable by a fine of 500 roubles, by $40-60 \mathrm{~km} / \mathrm{h}$ - the fine doubles and amounts to 1000 roubles, by 60 $80 \mathrm{~km} / \mathrm{h}-2500$ roubles, if the speed exceeds more than At $80 \mathrm{~km} / \mathrm{h}$, a fine of 5,000 roubles follows.

2.Departure to the lane intended for public transport. For the first time this violation began to be recorded by cameras in Moscow, where for public transport it was decided to allocate separate lanes. Then other subjects of the country joined the capital. Local authorities establish the days on which cars can leave for designated lanes (most often weekends), and also determine the types of vehicles that are equated with public (for example, taxis). In large cities - Moscow and St. Petersburg - for driving in a strip intended for public transport, a fine of 3,000 roubles follows, in other subjects the fine is 1,500 roubles.

3. Violation of the markup. The complexes are installed at the exits, corners, at the entrances to the tunnels and inside them. The fine is 500 roubles.

4. Roadside driving. Often in order to avoid traffic jams "reckless" drive to the sidelines. Thus, they create an even greater congestion on the road, trying to wedge themselves back onto the roadway. For this violation, a fine of 1,500 roubles is provided. 
5. Departure to a busy intersection. Drivers do not have time to cross the intersection at the traffic light allowing signal, appear in the middle of the road, thereby making it difficult for cars moving in the perpendicular direction. This causes traffic congestion in all directions. To identify violators, "waffle marking" was introduced at intersections. When the prohibition signal lights up and the car is inside this marking, the camera detects a violation, which will be followed by a fine in the amount of 500 to 1000 roubles.

6. Violations at pedestrian crossings. One of the most frequent and dangerous violations of drivers. Due to the high mortality rate due to non-admission of pedestrians, the fine for this violation was increased and ranges from 1,500 to 2,500 roubles. (5). The camera begins to follow the path of the pedestrian as soon as he steps on the crosswalk. At the same time, trajectories of all vehicles are recorded. In case of intersection of the paths of a pedestrian and a car, a violation will be recorded and, as a result, the driver will receive a fine.

7. The intersection of the stop line. Complexes relatively recently began to determine this violation. It is enough for the driver to cross the stop line with the front wheels, as the camera will immediately record a violation. The fine is 800 roubles, and if there is a corresponding sign in front of the stop line -500 roubles.

8. Access to the prohibition signal of traffic lights. The red and yellow traffic signals are prohibited. The complexes fixing this violation began to be installed not only on busy roads, but also at railway crossings. Even if the barrier at the crossing is raised, the driver needs to wait until the red lights of the traffic light go out. The fine is 1,000 roubles, and in the case of a repeated violation increases by five times.

9. Stop in a prohibited place. The latest versions of cameras have a viewing angle of 360 degrees, they can distinguish a car that has stopped in traffic from a standing at the curb, and also record the parking time of the vehicle. In Moscow and St. Petersburg, the fine for this violation is 3,000 roubles, in other cities of Russia - 2,000 roubles.

In addition, the technical means of photo-video recording should protect the information received in accordance with the legislation of the Russian Federation in the field of information and its protection [6]. The reliability of the data is confirmed by the used enhanced electronic signature [7].

The work of the complexes consists of several important stages:

1. At the initial stage, information is collected about vehicles traveling in the field of view of the camera. Occurring violations are recorded.

2. The received information about traffic violations is transmitted to the Center for Automated Fixing of Administrative Violations (abbreviated as CAFAP) of the GAI.

3. Data is accumulated and undergoes primary processing. There is an appeal to the database of the search and registered cars to supplement the previously obtained information [8].

4. Automatic analysis of information [9]. According to the data recorded by the cameras, a decision is made on the existence of an offense. A decision is issued to impose a fine, which is then sent to the owner of the vehicle.

The use of FVF complexes is regulated by the federal law "On Amendments to the Code of the Russian Federation on Administrative Offenses". The Code of Administrative Offenses of the Russian Federation says that the owner of the vehicle is responsible for the offense. The annex to the code also indicates that if the owner of the vehicle announced a review of the case, an additional verification of his whereabouts at the time the violation was committed is carried out [10]. If information about the impossibility of his stay in the car is confirmed, the owner is exempted from administrative responsibility [11].

In the places where the fixation systems are installed, the warning sign "Photofixation" must be put on and the appropriate road marking is applied. Such informing drivers helps to increase the vigilance of drivers and reduce the accident rate on the roads. 
Producers of fixation cameras constantly compete with each other, adding more and more features to their systems. Soon, cameras will record violations with unfastened drivers and passengers, as well as the facts of the absence of a compulsory insurance certificate for vehicles.

\section{Results}

The system of video recording violations of traffic rules is designed to automatically record violations of traffic rules and determine the facts of exceeding the speed of vehicles by different methods: radar, radarless (by video frames), by the value of the time interval and distance on a long stretch of road.

The system of video recording violations of traffic rules provides detection and fixing of the following types of traffic violations of the Russian Federation:

- passage to a prohibiting traffic signal in accordance with Art. 12.12. Part 1 of the Administrative Code of the Russian Federation;

- failure to comply with the stop requirement in front of the stop line in accordance with Art. 12.12. Part 2 of the Administrative Code of the Russian Federation;

- violation of the rules of passage through level crossings in accordance with Art. 12.10. Part 1 and Part 2 of the Administrative Code of the Russian Federation;

- movement along bicycle or pedestrian paths or sidewalks in accordance with Art. 12.15. Part 2 of the Administrative Code of the Russian Federation;

- departure in violation of the Rules of the Road to a lane intended for oncoming traffic or to tram tracks in the opposite direction in accordance with Art. 12.15. Part 4 of the Administrative Code of the Russian Federation;

- traffic in the opposite direction on the one-way road 12.16. Part 3 of the Administrative Code of the Russian Federation;

- non-admission of pedestrians at the regulated and unregulated pedestrian crossing art. 12.18 Administrative Code of the Russian Federation;

- $\quad$ vehicle stop or parking at a pedestrian crossing 12.19 part 3 of the Administrative Code of the Russian Federation;

- violation of the rules for stopping or parking the vehicle on the carriageway, where parking is prohibited by road signs or road markings;

- violation of the rules for stopping or parking the vehicle on the sidewalk;

- the location of the vehicle in the parking area in a prohibited way (for example, setting the vehicle is not parallel to the edge of the carriageway or in the second row on the carriageway);

- placement of transport centers, on the territory of parks, gardens, squares, boulevards, children's and sports grounds;

- violation of parking rules st.12.19 Administrative Code;

- intersection of the solid marking line of Art. 12.16 h. 1 4CoAP of the Russian Federation;

- vehicle passage under the prohibition sign of Art. 12.16 part 1 and part 3 of the Administrative Code of the Russian Federation;

- vehicle traffic in a lane for block vehicles 12.17 h. 1.1 Code of Administrative Offenses of the Russian Federation;

- turn left or turn in violation of the requirements prescribed by road signs or marking the carriageway art. 12.16 p. 2;

- violation of the rules for the location of the vehicle on the carriageway, oncoming traffic, as well as traffic along the roadsides or crossing an organized transport or pedestrian convoy or taking up a place in it $12.15 \mathrm{~h} 1$; 
- "Violation of parking rules closer than $50 \mathrm{~m}$ on both sides of level crossings", in accordance with clause 12.5 of the SDA of the Russian Federation, in accordance with Art. 12.19 part 1 of the Administrative Code of the Russian Federation;

- monitoring the traffic flow (counting the number of vehicles that traveled per unit time);

- $\quad$ search for vehicles located in the search bases in real time [11].

The system provides automatic photo fixation of vehicles that exceed the set speed threshold in the control zone in accordance with the Code of Administrative Offenses of the Russian Federation:

- $\quad$ excess of the established vehicle speed by more than 20 , but not more than 40 kilometers per hour (Art. 12.9 h. 2);

- $\quad$ exceeding the established vehicle speed by more than 40 , but not more than 60 kilometers per hour (Art. 12.9 h. 3);

- $\quad$ exceeding the established vehicle speed by more than 60 , but not more than 80 kilometers per hour (Art. 12.9 h. 4);

- $\quad$ exceeding the established speed of the vehicle by over 80 kilometers per hour (Art. 12.9 para. 5 ).

The composition of the system is determined by the contract and depends on the amount of recorded violations of traffic rules.

Structurally, the system can consist of control and photo radar controllers, which are the main elements of the system, GLONASS / GPS receivers, special software, IP video cameras and IR illuminators [11].

Information about traffic violations of the vehicle is transmitted to the data center (data processing center) via a secure GSM channel or other communication channels, in the data transfer formats of the agro-industrial complex VF.

Images received from IP video cameras are processed by controllers, and using special software, violations of the rules of the road are recorded and state registration plates (GRZ) of the vehicle violators are recognized. Then the STR allows you to make legible footage of violators and to provide information about violators through the communication channel in the form of receipts. To measure speed using the radar method, a photo radar controller and components for its placement and power supply are used. Using two registration kits (master and slave), which may include controllers, photo radar controllers, IP video cameras with IR projectors (depending on the system), the speed mode is fixed by the value of the time interval and distance [12].

To fix the speed mode by the radarless method (for video frames), an IP video camera and a control controller from the system or a photo radar controller are used or a control controller with a photo-fixing function (depending on the contract and the object of accommodation) by forming a sequence of video frames of the control zone with assigning to each video frame the current time and determining the distance traveled between the video frames in the control zone by special mathematical calculation.

The fixation of the speed mode can be determined in the control zone of the IP video camera by the radarless method, or between the registration sets by measuring the average speed by the value of the time interval and distance $[11,12]$.

The system of video recording of violations of the rules of the road is used to ensure safety on roads and to monitor compliance with traffic rules.

\section{Discussion}

An important component of the project is the justification of the need for its development and assessment of the effect of the implementation of the developed system. It is important to understand the benefits of automation and to recognize the effectiveness of work after 
process transformation. The effect of the development is to increase road safety by reducing the number of accidents. It is possible to predict the expected effect, depending on the installation sites of the complexes:

- the complex will reduce the number of accidents at a busy intersection by more than $20 \%$;

- the number of victims in road accidents will decrease by $10-40 \%$;

- $\quad$ the death toll drop by more than $30 \%$.

Expected results in financial and non-financial indicators, in comparison with analog solutions will allow:

- $\quad 10 \%$ reduction in labor input;

- $\quad$ increase in labor productivity by $10 \%$;

- improving the quality of work of operators by reducing routine operations;

- facilitating the work of operators due to the convenient and intuitive interface of the client module;

- $\quad 5 \%$ reduction in processing time;

- $\quad$ improving the reliability of data storage and their protection from various threats;

- $\quad$ speed of reaction to the occurrence of a violation;

- $\quad$ improving the stability of the complex by reducing the frequency of erroneous decisions;

- $\quad$ the speed of detecting errors in the operation of the complex is $5 \%$ higher;

- a high level of validity of decisions on violations by reinforcing them with reliable and timely information;

- improving the quality of statistical data analysis;

- etc.

\section{Conclusions}

In modern conditions of development of the transport system, with the increase in the number of cars, the risks of road traffic accidents increase. Automatic fixation complexes have great potential for the prevention and prevention of offenses in the field of traffic, the accident rate is significantly reduced due to increased discipline among vehicle drivers. In addition, such systems exclude subjective assessments and conflict situations between drivers and traffic police.

In this work:

- $\quad$ analysis of the current situation in the field of traffic was done;

- market analysis of existing means of fixing offenses was provided;

- the basic requirements for the created complex were defined;

- the functional structure of the system was designed;

- $\quad$ the functions of the complex were defined and described;

- the effect of the implementation of the developed system is determined [13-15].

The use of the complex will allow:

- measure vehicle speed without using radar from video frames;

- round-the-clock to record traffic violations at crossroads;

- receive signals from traffic lights;

- $\quad$ check the vehicle for the presence of an insurance policy;

- $\quad$ search the vehicle search bases;

- collect statistics;

- generate reports for the traffic police department;

- etc. 
A performance evaluation carried out at the final stage showed that the development of an information system is effective and will pay off in the shortest possible time. The created complex surpasses existing systems in accuracy and data reliability.

\section{References}

1. V. Volkova, G. Gorelova, N. Pankratova, 2020 IEEE 2nd International Conference on System Analysis and Intelligent Computing, SAIC 2020, 9239213 (2020)

2. O.A. Konovalova, Y.R. Nurulin, S.G. Redko, Proceedings - 2020 International Russian Automation Conference, RusAutoCon 2020 9208132, 93-97 (2020)

3. V.N. Volkova, V.N. Kozlov, V.E. Mager, L.V. Chernenkaya, Proceedings of 2017 20th IEEE International Conference on Soft Computing and Measurements, SCM 2017 7970533, 183-186 (2017)

4. A.A. Denisov, Introduction to information systems analysis (LPI, Leningrad, 1980)

5. A.A. Denisov, Modern problems of system analysis (2008)

6. M. Bolsunovskaya, S. Shirokova, A. Loginova, M. Uspenskij, IOP Conference Series: Materials Science and Engineering (2019) https://doi.org/10.1088/1757899X/497/1/012024

7. A. Wildani et. al., Proc. IEEE International Symposium on Modeling, 1-11(2009)

8. R.E. Kalman, Journal of Basic Engineering 82, 35-40 (1960)

9. M.B. Uspenskiy, S.V. Smirnov, A.V. Loginova, S.V. Shirokova, Proceedings of 2019 3rd International Conference on Control in Technical Systems, CTS $2019 \mathbf{8 9 7 3 2 4 5 ,}$ 11-14 (2019)

10. M.Yu. Ermolenko, Integrated business planning as minimization of uncertainty through multi-level planning (Russian Academy of Sciences, 2009) https://elibrary.ru/item.asp?id=26325728

11. M.V. Bolsunovskaya, A.V. Leksashov, A.V. Loginova, S.V. Shirokova, E3S Web of Conferences 91, 07011 (2019)

12. S.M. Aleksei, V.B. Marina, Advances in Intelligent Systems and Computing 1041, 171-178 (2020)

13. O.G. Kantor, S.I. Spivak, Informatics and its application 8(2), 111-121 (2014)

14. V.N. Volkova, G.V. Gorelova, A.A. Efremov et. Al., Systems and processes modeling (Yurait Publ., Moscow, 2016)

15. V.N. Volkova, Theory of information systems. Textbook (Saint-Petersburg Polytechnic University Publ., St. Petersburg, 2014) 